\title{
The Generalized Serial Test Applied to Expansions of Some Irrational Square Roots in Various Bases*
}

\author{
By W. A. Beyer, N. Metropolis and J. R. Neergaard
}

\begin{abstract}
A brief summary is given of the application of the generalized serial test for randomness to the digits of irrational $\sqrt{ } n$ in bases $t$ where $2 \leqq n, t \leqq 15$. The results are consistent, except for a few aberrations, with the hypothesis of randomness of the digits.
\end{abstract}

This is a brief report on the application of the generalized serial test (see [1]) for randomness to the expansions of some irrational $\sqrt{ } n$ in various bases. It can be considered as an extension of the work Good and Gover [1] have done with 10,000 binary digits of $\sqrt{ } 2$. I. J. Good (private communication) has remarked that some of the tests used in [4] are special cases of the generalized serial test.

The conclusion resulting from this study is that the results, except for a few aberrations given in Table 3, are consistent with the hypothesis of randomness of the digits of the square roots investigated. Since a total of about 2400 tests were made, perhaps the aberrations are not surprising, if one notes that $2400^{-1}=.0004$.

For completeness, a recapitulation is given (from [1]) of the generalized serial test for randomness as used here. Let a sequence of $N$ digits in base $t(t=2,3, \cdots)$ be given and let the sequence be circularized; i.e., the last digit is considered as being followed by the first digit. Let $n_{I}$ be the number of occurrences of the $\nu$-plet $I=$ $\left(i_{1}, i_{2}, \cdots, i_{v}\right)$ in the circularized sequence. Define

and

$$
\psi_{0}^{2}=\psi_{-1}^{2}=0, \quad \psi_{v}^{2}=\frac{t^{\nu}}{N} \sum_{I}\left(n_{I}-\frac{N}{t^{\nu}}\right)^{2},
$$

$$
\nabla^{2} \psi_{\nu}^{2}=\psi_{\nu}^{2}-2 \psi_{\nu-1}^{2}+\psi_{\nu-2}^{2}
$$

for $\nu \geqq 1$. The distributions $\nabla^{2} \psi^{2}$, are asymptotically chi-square with the number of degrees of freedom equal to $t^{\nu}-2 t^{\nu-1}+t^{\nu-2}$.

The authors [3], [4] have computed $N$ digits of the fractional part of $\sqrt{ } n$, base $t$, for $n=2,3,5,6,7,10,11,13,14$, and 15 in accordance with Table 1 .

This was accomplished by computing $88064=43 \cdot 2^{11}$ binary digits of the fractional part of $\sqrt{ } n$ and then changing base. In the conversion from binary to base $t$, it can be shown that $N=2^{11}[43 \log 2 / \log t]$ digits are accurate, where [ ] denotes largest integer. (Note that for $t=6, N=2^{15}$.) Because of some minor technical difficulties, the last 1 or 2 digits may not be accurate. For this reason 88062 replaces 88064 in the title of [3].

Received September 29, 1969, revised December 29, 1969.

AMS Subject Classifications. Primary 1050; Secondary 1009, 6299.

Key Words and Phrases. Serial test, generalized serial test, statistics of square-root digits, square roots, square roots in several bases, expansions of square roots, random sequences, statistical study of digit sequences.

* Work performed under the auspices of the U. S. Atomic Energy Commission.

Copyright $\odot$ 1971, American Mathematical Society 


\begin{tabular}{r|c||c|c}
\hline$t$ & $N$ & $t$ & $N$ \\
\hline 2 & 88046 & 11 & 24576 \\
3 & 55296 & 12 & 22528 \\
5 & 36864 & 13 & 22528 \\
6 & 32768 & 14 & 22528 \\
7 & 30720 & 15 & 20480 \\
10 & 24576 & & \\
\hline
\end{tabular}

TABLE 1. Number of computed digits of $\sqrt{ } n$ in base $t$.

The quantity $\nabla^{2} \psi^{2}$, is computed for each whole block of length $x \cdot 1000$ of $(\sqrt{ } n)_{1}$; i.e., for the blocks with digits: $1 \rightarrow x \cdot 1000,(x \cdot 1000+1) \rightarrow 2 x \cdot 1000$, etc., and also for the entire sequence of $N$ digits of $(\sqrt{ } n)_{1}$. The values of $\nu$ and $x$ selected are listed in Table 2.

This paper will list only the aberrations observed; if the chi-square level (tail area) is less than .007 , it is listed in Table 3. The remainder of the data has a $\chi^{2}$ level greater than .007 .

It should be noted that with the exception of $\left((13)^{1 / 2}\right)_{12}$, the aberrations occur in the intermediate digits and disappear when a larger sample is examined.

Remark. Since the work of Good and Gover [1] is referred to several times, it should also be mentioned that their suggestion for calculating $\sqrt{ } 2$ (or $\sqrt{ } m$ in [2]) is generalized in [5].

Acknowledgment. The computations were made on the MANIAC II computer in our laboratory.

Appendix. Certain errors are noted in the report of the values of $\nabla^{2} \psi^{2}$ $(1 \leqq \nu \leqq 10)$ for 10,000 binary digits as given in Table 1 of Good and Gover [1]. These errors apparently arose from two sources: rounding error in the value of $\nabla^{2} \psi^{2}$, itself and the fact that their 10,000th binary digit was obtained as 0 , whereas in fact it is a l. For $\nu=8$, block 5 should be 37.2 and block 8 should be 78.8. The entries in block 10 should be replaced by the sequence (starting with $\nu=1$ ): $1.3,0.0,0.7,10.3$,

\begin{tabular}{c|l|r}
\hline \multicolumn{1}{c|}{$t$} & \multicolumn{1}{|c|}{$\nu$} & $x$ \\
\hline 2 & $1,2, \ldots, 10$ & 10 \\
3,5 & $1,2,3,4$ & 10 \\
$6,7,10$ & $1,2,3$ & 5 \\
$11,12,13,14,15$ & 1,2 & 5 \\
\hline
\end{tabular}

TABLE 2. Values of $\nu$ and $x$ for the various bases $t$. 


\begin{tabular}{r|r|r|c|l}
\hline$n$ & \multicolumn{1}{c|}{$t$} & $\nu$ & Block & Level \\
\hline 2 & 2 & 2 & $10001 \rightarrow 20000$ & .0058 \\
2 & 14 & 1 & $20001 \rightarrow 25000$ & .0053 \\
3 & 3 & 1 & $10001 \rightarrow 20000$ & .00025 \\
3 & 7 & 1 & $20001 \rightarrow 25000$ & .0015 \\
5 & 15 & 1 & total & .004 \\
7 & 7 & 1 & $25001 \rightarrow 30000$ & .006 \\
10 & 5 & 2 & $30001 \rightarrow 40000$ & .005 \\
11 & 5 & 2 & $10001 \rightarrow 20000$ & .0066 \\
13 & 3 & 2 & $40001 \rightarrow 50000$ & .00027 \\
13 & 12 & 1 & total & .0015 \\
14 & 6 & 2 & $15001 \rightarrow 20000$ & .0023 \\
\hline
\end{tabular}

TABLE 3. Aberrations.

$6.1,16.6,22.1,67.2,130$, and 236 . The entries in the block marked "whole" should read: $.4, .5,3.4,2.6,9.1,19.8,51.5,58.9,128,242$. The four which are boldface were correct in their original table.

University of California

Los Alamos Scientific Laboratory

Los Alamos, New Mexico 87544

1. I. J. Good \& T. N. Gover, "The generalized serial test and the binary expansion of $\sqrt{ } 2$," J. Roy. Statist. Soc. Ser. A, v. 130, 1967, pp. 102-107. p. 434.

2. I. J. GOOD \& T. N. GOVER, "Corrigendum," J. Roy. Statist. Soc. Ser. A, v. 131, 1968,

3. W. A. Beyer, N. Metropolis \& J. R. Neergaard, "Square roots of integers 2 to 15 in various bases 2 to 10: 88062 binary digits or equivalent," Math. Comp., v. 23, 1969, p. 679. RMT 45.

4. W. A. Beyer, N. Metropolis \& J. R. Neergaard, "Statistical study of digits of some square roots of integers in various bases," Math. Comp., v. 24, 1970, pp. 455-473.

5. C. J. Everett \& N. Metropolis, "Approximation of the $\nu$ th root of $N$," Discrete Mathematics. (To appear.) 